MODELING, IDENTIFICATION AND CONTROL, 2003, VOL. 24, NO. 1, 3-13

doi:10.4173/mic.2003.11

\title{
Wave Detection and Characterization from Current and Voltage Signals of an Aluminium Smelting Cell $\dagger$
}

\author{
INGAR SOLBERG*
}

Keywords: wave analysis, aluminium production, signal processing, visualisation. anode current, electrolysis

In aluminium electrolysis cell there is a layer of $10-20 \mathrm{~cm}$ of liquid aluminium. On top of this there is a layer of a melted salt electrolyte with a number of carbon anode blocks partially submerged. The density difference of the two liquids is small and low frequency waves can occur in the interface. The waves cause variation in the current distribution between the individual anode carbon blocks. These variations are in this article analysed and visualised with MATLAB to show information of the temporal variations, frequencies and spatial modes of the waves.

\section{Introduction}

The purpose of this paper is twofold:

1. Show how a matrix calculation program as MATLAB $^{\mathrm{k}}$ can be applied to calculate and visualise wave phenomena from measurements.

2. Show an analysis of anode current measurements that might be useful for verifying mathematical models and for control of aluminium smelting cells.

An aluminium smelting cell is like a large bath tube with an area of $10-35 \mathrm{~m}^{2}$. In the bottom of the cell there is a layer of $10-20 \mathrm{~cm}$ of liquid aluminium. On top of this there is a layer of a melted salt mixture (commonly called bath). Partially submerged into the bath are 12-30 carbon blocks. Electrochemically the liquid metal acts as the cathode, the salt as the electrolyte and the carbon blocks as the anode.

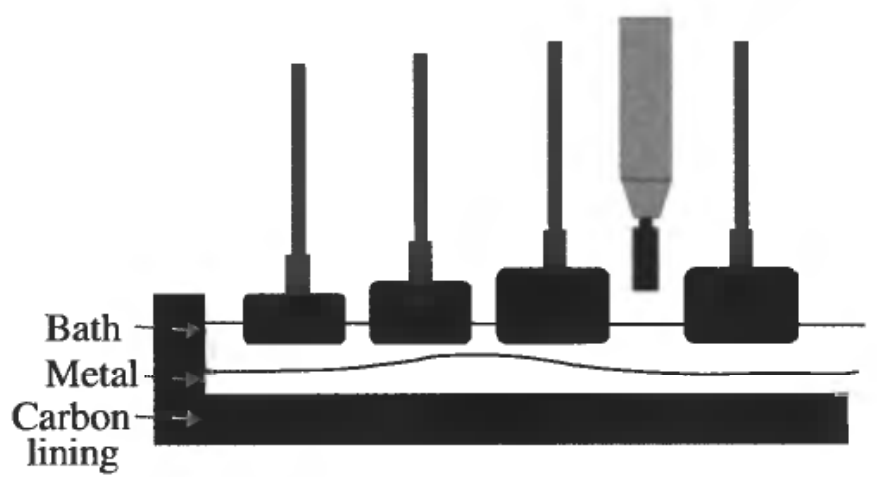

Figure 1. Cross-section of cell.

*Elkem Aluminium Mosjøen, NO-8655 Mosjøen. Tel: +4775179152. Email: ingar.solberg@, elkem.no

$\dagger$ An early version of this article was presented at Nordic Matlab Conference 2001. October 2001, Oslo, Norway. 


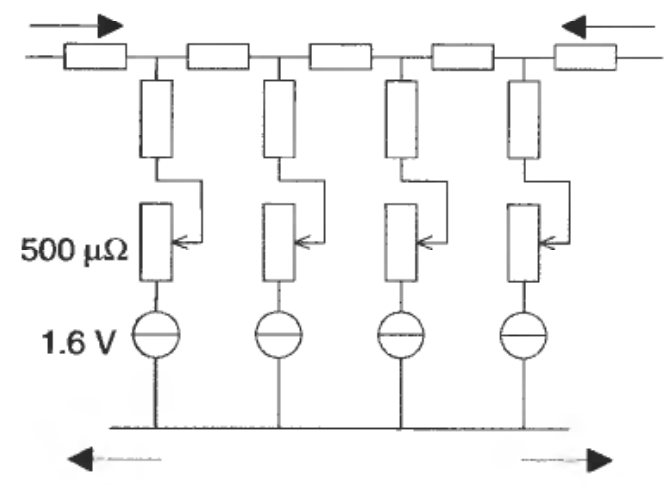

Figure 2. Electrical equivalent.

The cell current of 100 to $300 \mathrm{kA}$ causes a strong magnetic field. The current flowing through the liquids in this field generates forces that form the bath/metal interface and causes liquid flow. A sufficient circulation of the bath and a stable bath/ metal interface is important for a good operation of the cell.

The density difference of the two liquids is small and low frequency waves can occur at the interface. These waves will cause variation in the distance between the liquid metal and the underside of the individual anode blocks. This is commonly named the AC-distance (Anode-Cathode distance). The anode blocks are electrically coupled in parallel, and the resistance of the bath layer dominates any other resistive elements in the circuits. Thus variations in the AC-distance causes variation in the current distribution between the anode blocks. The total current is kept almost constant by the rectifier controller.

An electrical equivalent diagram of a cell with four anodes is shown in Figure 2. Currents enter from both sides at the top and leave to both sides at the bottom. The voltage sources are $1.6 \mathrm{~V}$. The variable resistors represent the bath and are much larger than the others, which are ignored in this article.

Mathematical models have been set up mainly based on Maxwell's equations, Navier-Stoke's equation, Ohm's law and the equation for magnetic force $(\mathrm{d} \mathbf{F}=\mathbf{j d} \mathbf{V} \times \mathbf{B})$. Mostly they have been used to find static solutions for flow and interface geometry. With more powerful computers, linear dynamic models are also used to predict the stability of cells, and how the electrical current and magnetic field affects stability (Romerio and Antille (2000)). Full dynamic simulation of the nonlinear model is very demanding.

Keniry, Barber, Taylor and Welch (2001) shows some other analysis of anode current measurements and references to other works in this area.

The present paper does not involve any dynamic model, but only mathematical analysis of measurements from real cells. The frequencies and wave modes obtained can be compared to those predicted by the model calculations.

\section{Measurements}

All data in this article are from similar cells at Elkem's plant in Mosjøen, Norway (Figure 3). The cells run at a current of 155-160 kA. They have 2 rows of 9 anode blocks each, which are numbered as in Figure 12.

Each anode has a copper stem that is connected to a common bus with a separate 


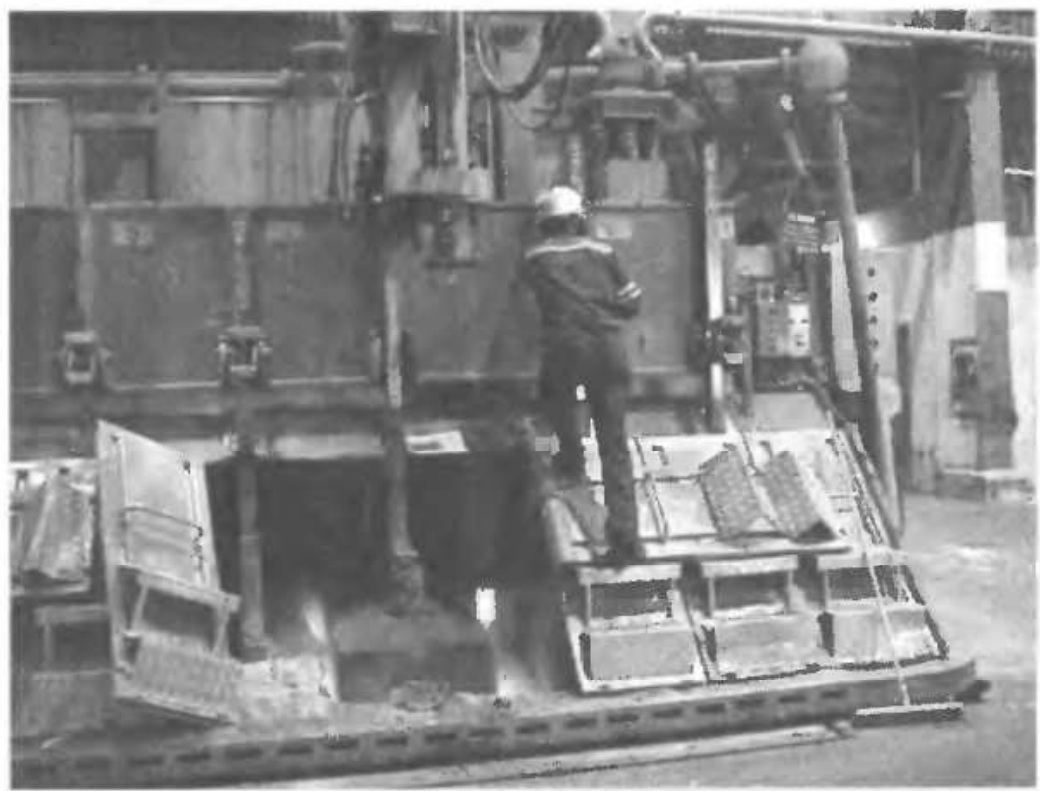

Figure 3. Cell in Mosjøen.

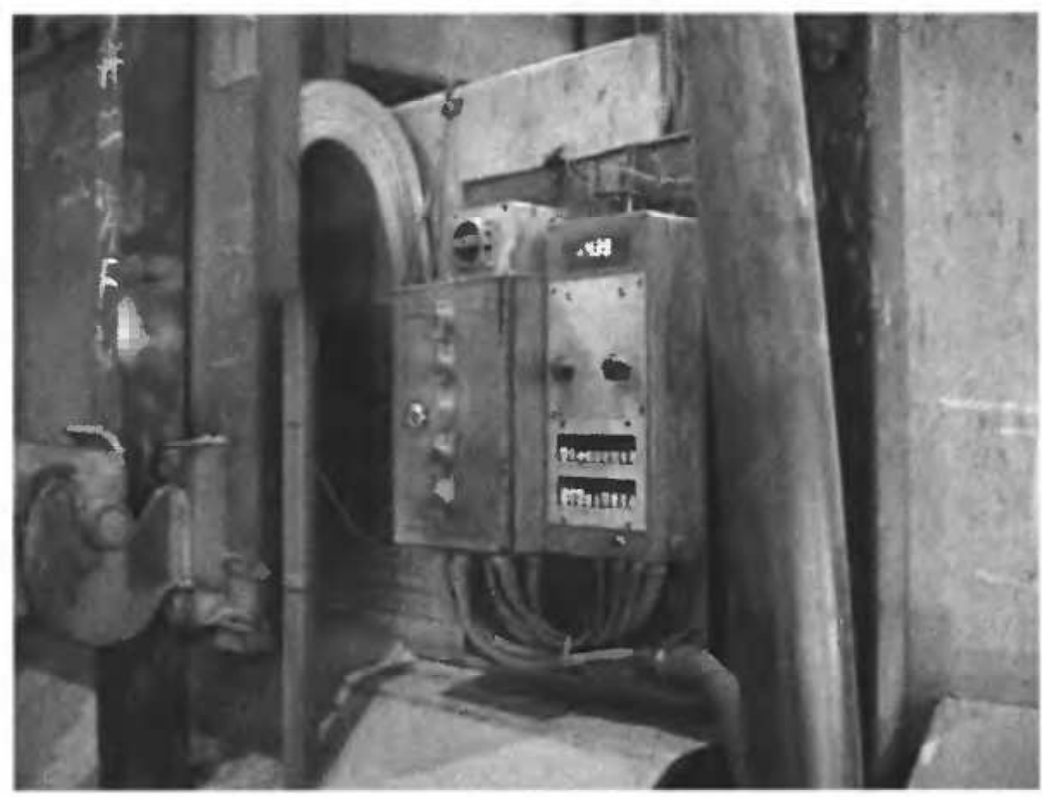

Figure 4. Flexible connection and display unit.

flexible of aluminium sheets (Figure 4). To find the current passing through each anode the voltage drop over each flexible is measured. The total voltage for the cell and its electrical buswork is also measured.

All these signals are wired up to a display unit at each cell. To collect data a logger was connected to a bus within this unit. Sampling interval used was in the range $1 \mathrm{~s}$ to $15 \mathrm{~s}$. 


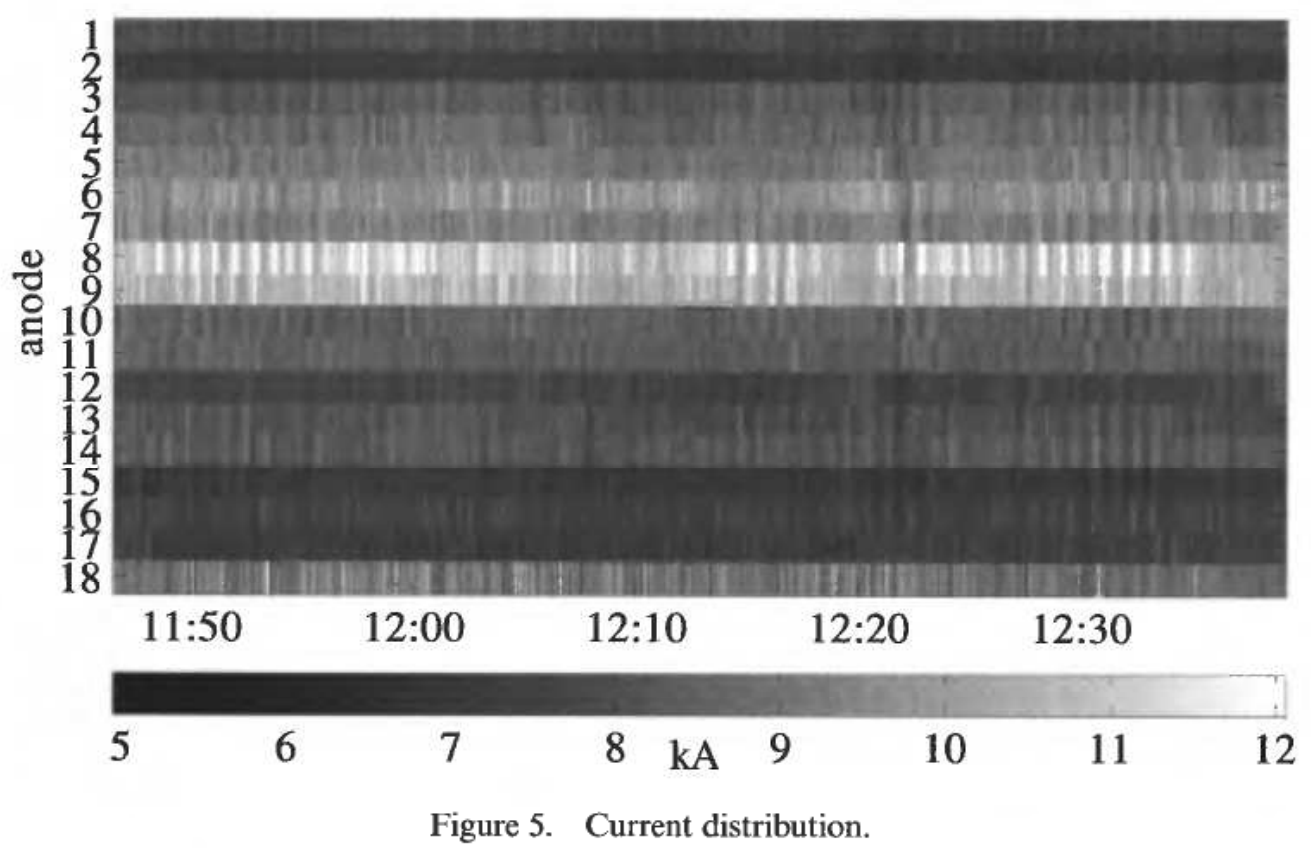

The data were transferred from the logger, transformed to physical units and then sent to Matlab as matrices with 20 columns: Time, 18 anode currents and cell voltage.

\section{Visualization and Analysis}

Raw data

The 18 anode currents can be visualised with a graph function like imagesc, where a colour scale is used to show the current through each anode as functions of time (Figure 5).

As is easily seen there is a large variation in the average current through each anode, and some vertical 'ribs' that may be caused by waves. To get a better display of these 'ribs' the average current could be subtracted for each anode. We will however first apply another transformation commonly used in control systems for these cells:

$$
R=\left(U-U_{0}\right) / I
$$

where $U$ is the cell voltage, $U_{0}$ is a fixed voltage of about $1.6 \mathrm{~V}$ and $I$ is the current passing through the cell. The calculated $R$ is called pseudo-resistance. For the whole cell $R$ is relatively insensitive to variations in the current through the cell, and almost proportional to the average AC-distance. Applying the same transformation to the anodes using their individual currents we get a 'measurement' of the AC-distance under each anode and thus the position of the bath/metal interface.

\section{Process considerations}

The main reaction of the cell is

$$
2 \mathrm{Al}_{2} \mathrm{O}_{3}+3 \mathrm{C} \rightarrow 4 \mathrm{Al}+3 \mathrm{CO}_{2}
$$




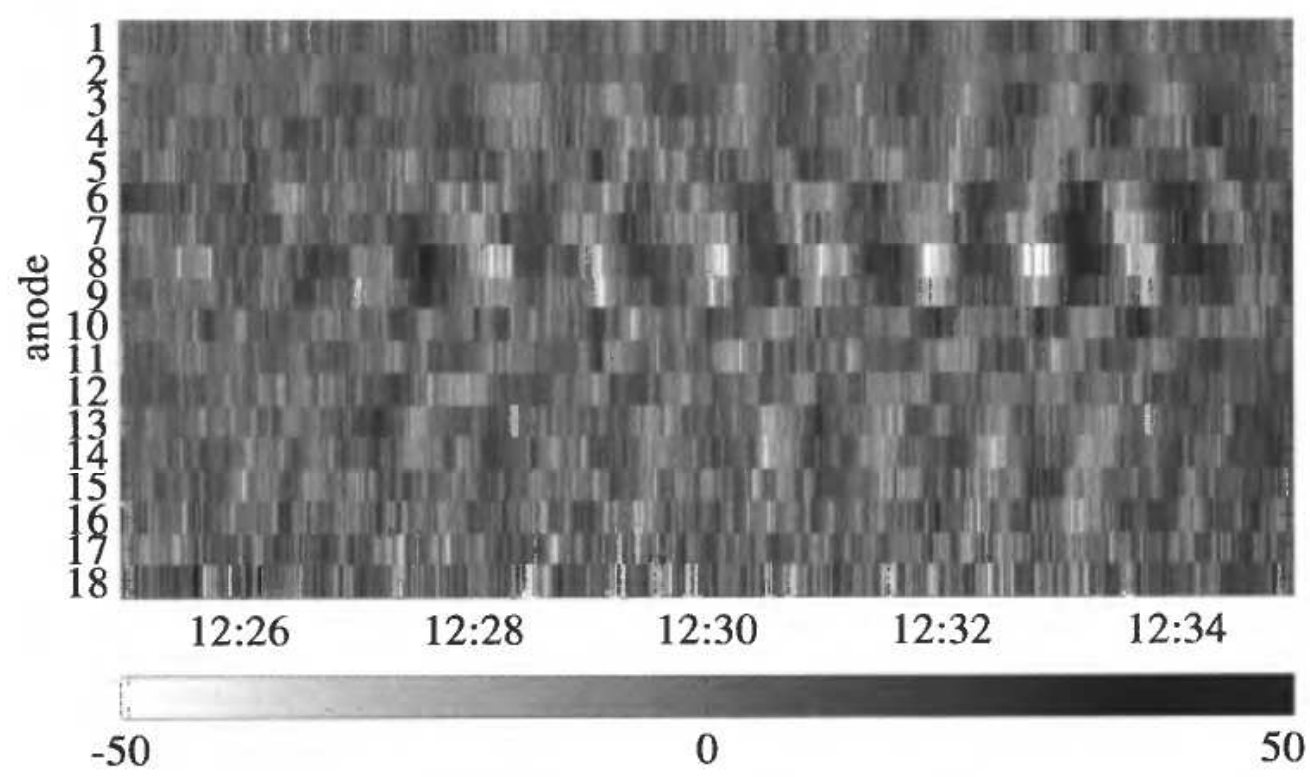

Figure 6. Resistance variations.

The carbon comes from the anodes that are consumed by the process at a rate of about $15 \mathrm{~mm} /$ day (depending on the current density at the surface). This means that consumed anodes have to be replaced after 30 days. There is a schedule for this distributing the replacements evenly over this 30 days period. After a new cold anode has been installed it takes about 8 hours before the bath that froze on its surface has melted. Normally a new anode is set so that its underside is some $\mathrm{mm}$ higher than that of the anode replaced. Thus it takes a little less current and is consumed at a lower rate than its neighbours. This reduces the differences in AC-distance with time. Unfortunately another anode has to be replaced before a steady state is reached so an even AC-distance and current distribution is never achieved.

We are interested in short-term variations, so the average and linear trend in is removed for each anode (by dtrend). Doing this we are left with variations around zero. Figure 6 shows the result of these transformations applied to an interval of the data from Figure 5. A wave with a period of about 50 s is clearly visible. We can also see that when the resistance of anode 8 is high, that of anode 10 is low.

The $\mathrm{CO}_{2}$ gas will accumulate as bubbles under the anodes. The build up and release of bubbles causes resistance variations (noise) of about $0.2 \mathrm{~Hz}$ and above which is also showing up in the graph.

\section{Time-frequency analysis}

To be able to look for waves in a larger set of data a time-frequency plot can be made. The Gabor wavelet transform can be used for this (from Mallat (1998)). This transform is a convolution of the signal with

$$
\psi_{\omega, \sigma}(t)=\frac{1}{\sqrt{2 \pi \sigma}} e^{j \omega t} e^{-t^{2} / 2 \sigma^{2}}
$$

at frequencies $\omega$ and with time window widths $\sigma$. A logarithmic spacing of the frequencies is used, and $\sigma$ is chosen inverse proportional to the frequency spacing. 


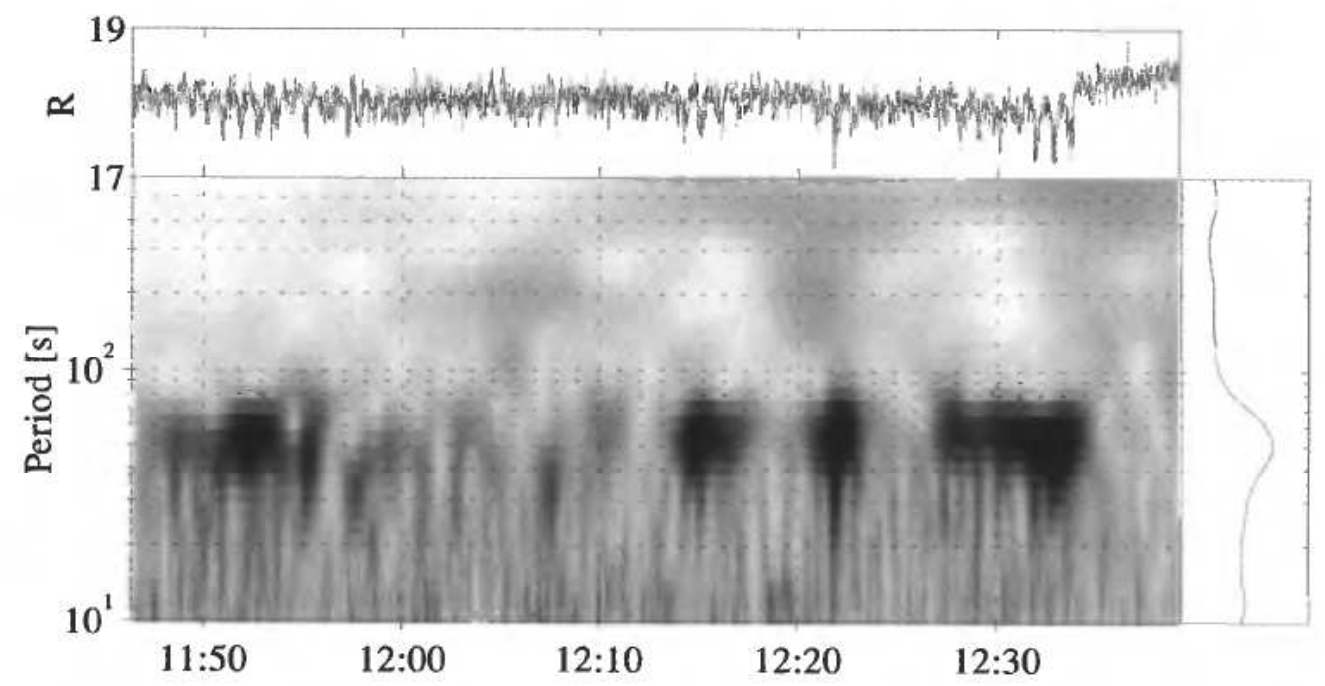

Figure 7. Time-frequency plot with low frequency resolution.

As convolution in time space is equivalent to multiplication in frequency space an $f f t$ of $R$ and $\psi$ is done followed by an element-by-element multiplication and an ifft of the result. The squared absolute values for each anode are then added up.

The time-frequency plot in Figure 7 shows some dark spots indicating waves with a period of about 50 seconds. The graph at the top shows the total resistance for the cell, while that on the right shows the total frequency distribution. Far right in the time interval shown the AC-distance has been increased by the control system. Normally this improves the stability (as here) and also increases the energy consumption.

It's possible to get a higher frequency resolution by specifying more frequencies. Eventually higher frequency resolution gives lower time resolution.

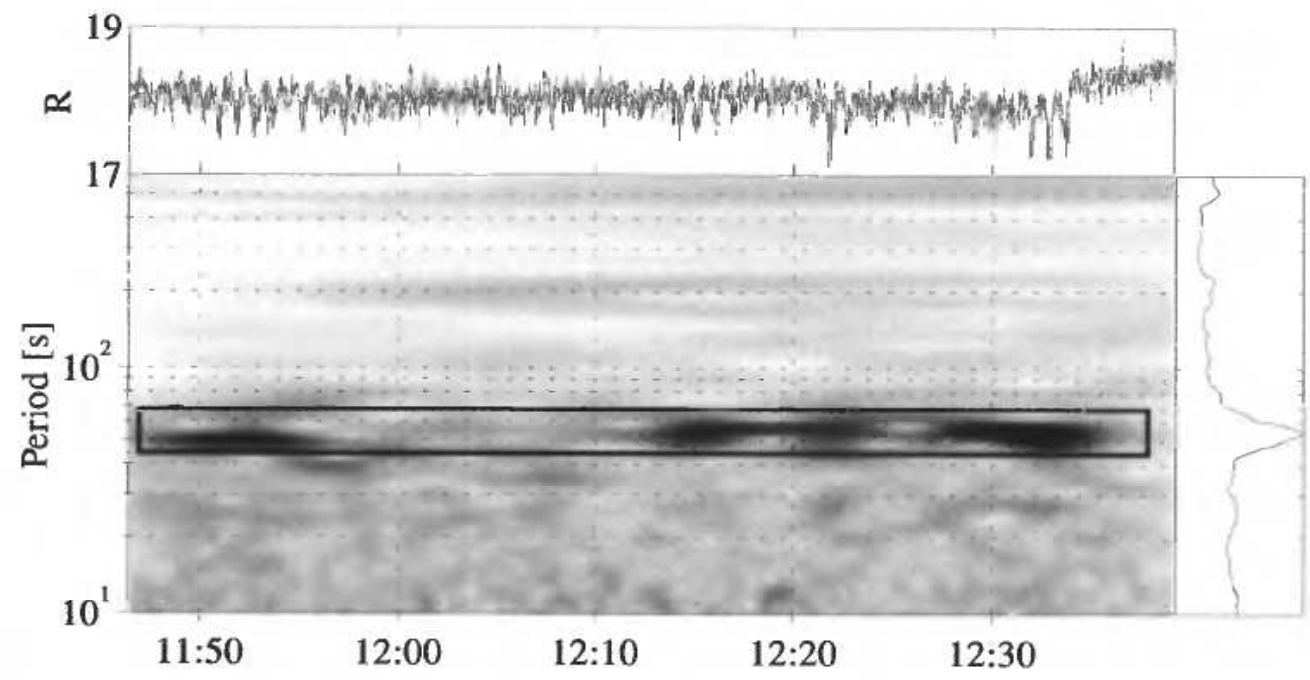

Figure 8. Time-frequency plot with high frequency resolution. 

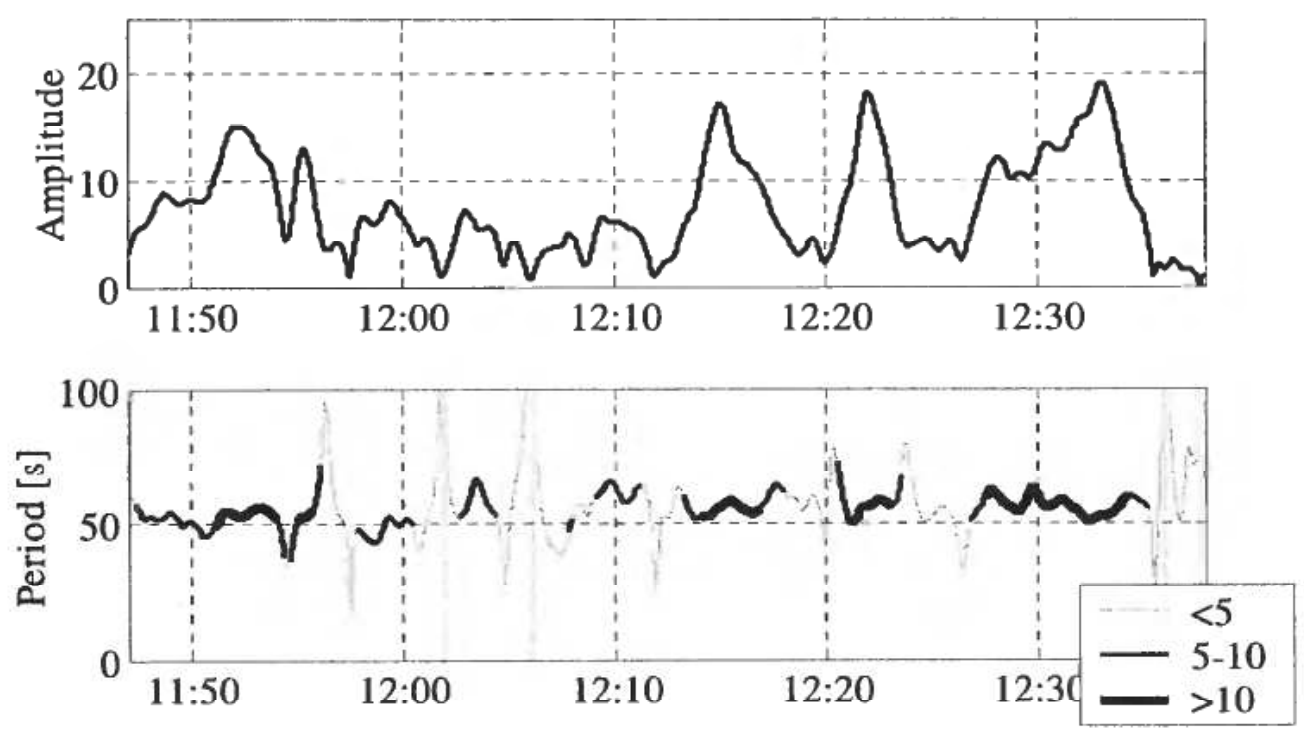

Figure 9. Amplitude and period variation.

Spatial wave mode and time variation

Now it's time to se how the wave looks like that's causing the black spots in the graphs above. The following procedure has been implemented:

1. On the plot, mark with a rectangle the time and frequency range of interest. (As done Figure 9.)

2. Convolute $R$ of each anode with $\left(\psi_{\omega, \sigma}(t)\right.$ where $\omega$ is at the centre of the rectangle and $\sigma$ is inverse proportional to its frequency width. The result is a complex band-limited signal from each anode.

3. Organise the complex signals as a matrix $\mathbf{D}$ with as many columns as there are anodes. Then try to fit $\mathrm{D}$ with the outer product $\mathbf{w p}^{H}$, where $\mathbf{w}$ is a complex vector representing the wave as a function of time, and $\mathbf{p}$ is another complex vector representing the relative amplitude and phase of $R$ at each anode. A least squares solution is found minimising the Frobenius norm of the residue by minimising

$$
f(\mathbf{w}, \mathbf{p})=\frac{1}{2}\left\|\mathbf{w} \mathbf{p}^{H}-\mathbf{D}\right\|_{F}^{2}=\frac{1}{2} \operatorname{tr}\left(\left(\mathbf{w} \mathbf{p}^{H}-\mathbf{D}\right)^{H}\left(\mathbf{w} \mathbf{p}^{H}-\mathbf{D}\right)\right)
$$

To normally get a unique solution the element of $\mathbf{p}$ corresponding to the column of $\mathbf{D}$ with the largest norm is fixed to unity. This column of $\mathbf{D}$ is also used as initial value for $\mathbf{w}$ when searching for the solution.

4. Searching methods are applied to the problem taking advantage of its special structure. See appendix 1.

The amplitude and period of the fitted $w$ is shown in Figure 9. The line weight in the period plot is larger for the larger amplitudes, when estimation is more reliable.

The average period seems to be a little more than 50 seconds.

The spatial wave mode $\mathbf{p}$ can be visualised as a polar plot of its complex entries 


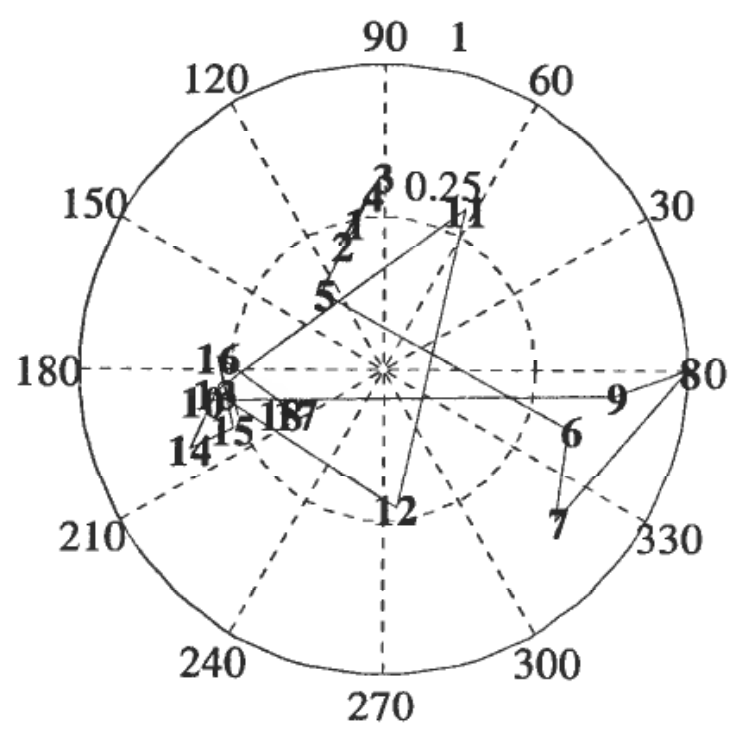

Figure 10. Amplitude and phase at the anodes for the wave mode.

with annotation of the anode numbers as in Figure 10. (The square root of the amplitude is shown to improve the visibility of the smaller amplitudes.)

Another option is to use one graph for amplitude and one for the phase as in Figure 11.

This shows that the wave has its maximum at anode 8 , and that the resistance of the neighbouring anodes 6,7 and 9 are almost in phase with it.

With colours available it's possible to code phase as hue and amplitude as value in the HSV colour model. Without colour a vector plot is used shoving anode layout
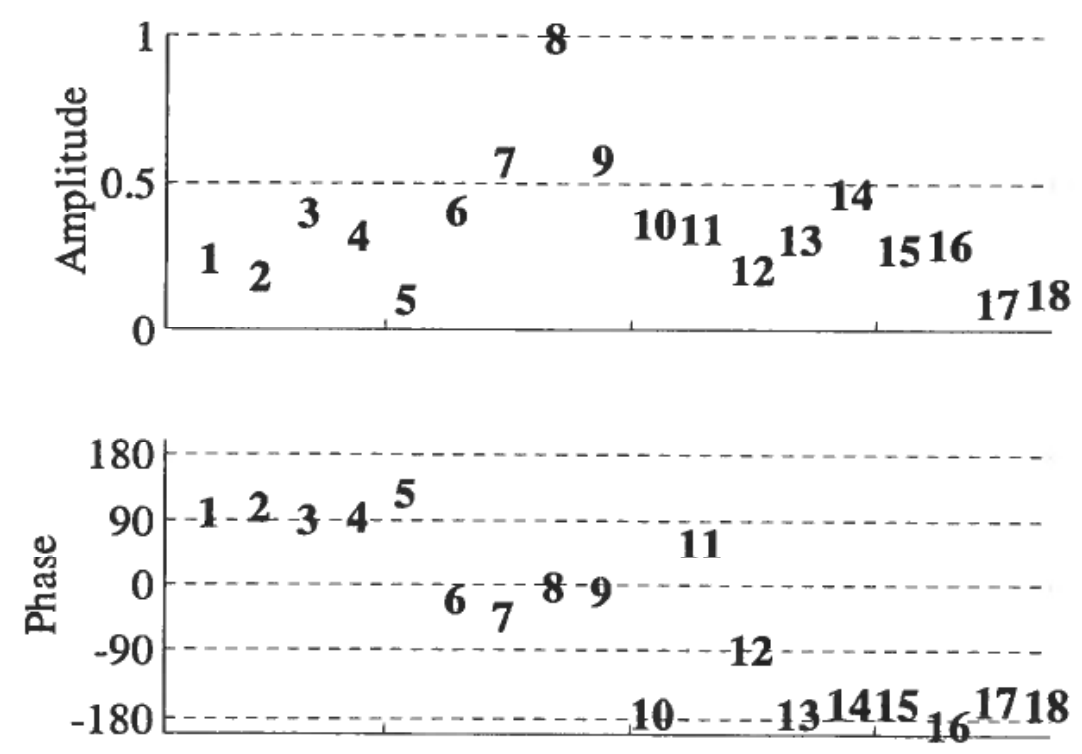

Figure 11. Amplitude and phase at the anodes for the wave mode. 


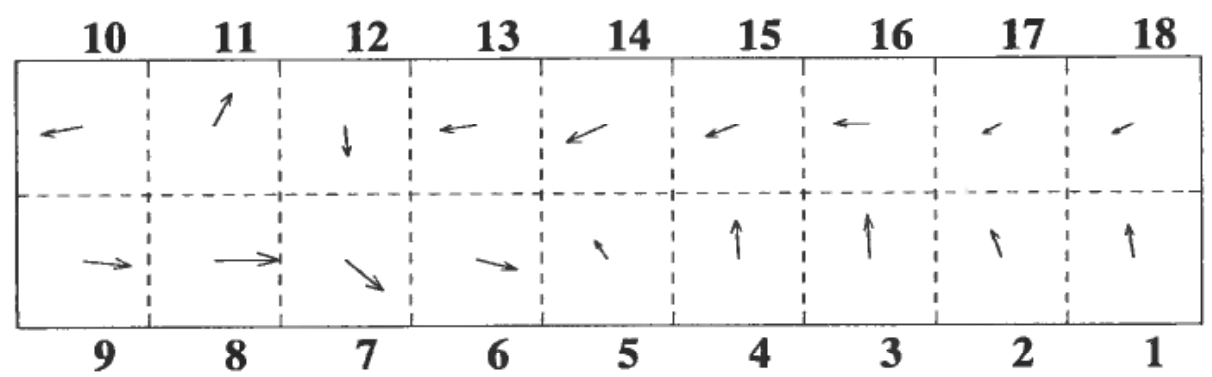

Figure 12. Vector plot of spatial distribution of amplitude and phase (top view).
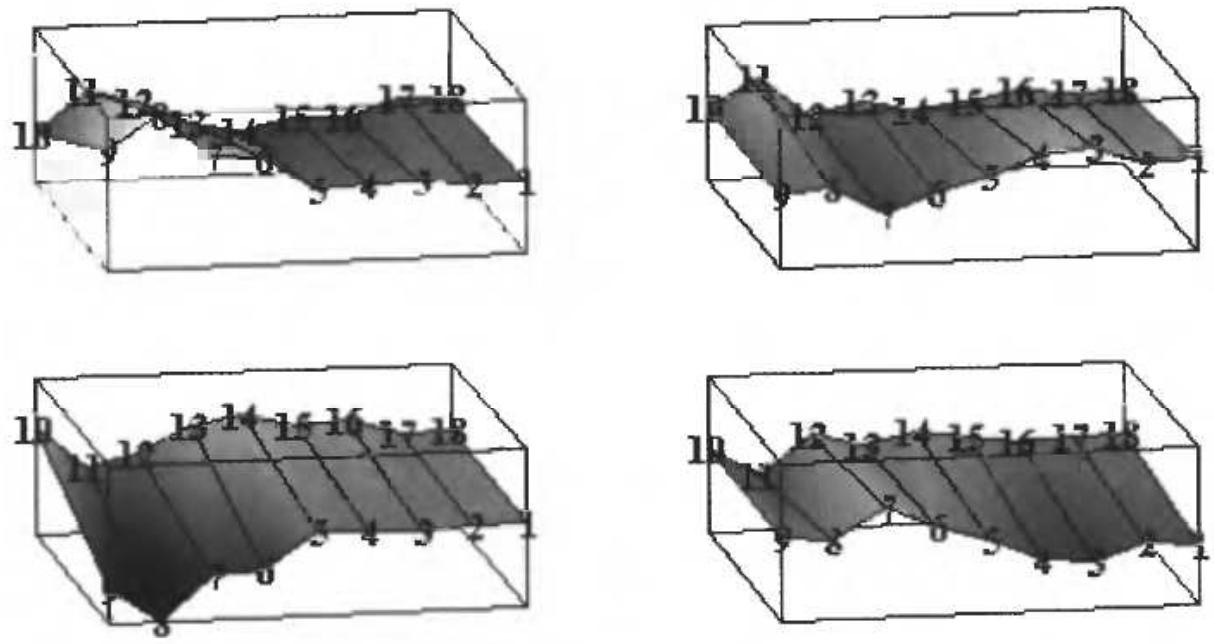

Figure 13. Wave propagation.

and the phase and amplitude in Figure 12. As in Figure 10 the square root if the amplitude is shown to make the smaller vectors more visible.

With the ability to show small movies the waves can be displayed in an even better way. Here are four plots 0.25 period apart (the real, imaginary, -real and -imaginary parts of $\mathbf{p}$ ).

\section{Future Applications}

Few or may be no plant has all their anode currents available on line for control and analysis today. With the reduced cost of the equipment taking these measurements and constant pressure for squeezing more metal out of the cells by increasing the current this may change in the near future. Analysing these currents may then be an affordable tool to diagnose problems at cells and for improving the control.

\section{Summary}

Some ways using Matlab to find and analyse waves in aluminium smelting cells have been shown. Time-frequency analysis with retention of the phase information 
makes it possible to find both the spatial wave modes and the variation with time. They may be compared to predictions from mathematical models for their verification.

There are a number of ways to display wave phenomena, of which a few suitable on paper have been shown.

\section{Acknowledgements}

The author wants to acknowledge his employer Elkem Aluminium for giving him the time and permission to publish these results and the pictures from the plant in Mosjøen.

\section{Appendix}

The least squares solution is found by minimising the Frobenius norm

$$
\|A\|_{F}=\sqrt{\Sigma \Sigma\left|a_{i j}\right|^{2}}
$$

by searching for the minimum of

$$
f(\mathbf{w}, \mathbf{p})=\frac{1}{2}\left\|\mathbf{w} \mathbf{p}^{H}-\mathbf{D}\right\|_{\mathbf{F}}^{2}
$$

The first order conditions then are

$$
\left[\begin{array}{ll}
\nabla_{p} f & \nabla_{w} f
\end{array}\right]=\left\lfloor\mathbf{w}_{H}\left(\mathbf{w} \mathbf{p}^{H}-\mathbf{D}\right) \mathbf{p}^{H}\left(\mathbf{w p}^{H}-\mathbf{D}\right)^{H}\right\rfloor=\mathbf{0}^{H}
$$

and the Hessian is

$$
\left[\begin{array}{cc}
\|\mathbf{w}\|_{2}^{2} \mathbf{I} & 2 \mathbf{p} \mathbf{w}^{H}-\mathbf{D}^{H} \\
2 \mathbf{w p} \mathbf{p}^{H}-\mathbf{D} & \|\mathbf{p}\|_{2}^{2} \mathbf{I}
\end{array}\right]
$$

Alternating Newton steps in the subspaces of $\mathbf{p}$ and $\mathbf{w}$ can be applied with

$$
\Delta \mathbf{p}=\frac{-\left(\mathbf{w} \mathbf{p}^{H}-\mathbf{D}\right)^{H} \mathbf{w}}{\|\mathbf{w}\|_{2}^{2}}
$$

and

$$
\Delta \mathbf{w}=\frac{-\left(\mathbf{w p} \mathbf{p}^{H}-\mathbf{D}\right) \mathbf{p}}{\|\mathbf{p}\|_{2}^{2}}
$$

The steps in each subspace are exact so no line searches are required. The procedure is simple and robust, but convergence is linear and may be slow.

Alternatively a Newton step $\left[\begin{array}{ll}\Delta \mathbf{p}^{H} & \Delta \mathbf{w}^{H}\end{array}\right]^{H}$ in the full search space is calculated from the equation

$$
\left[\begin{array}{cc}
\|\mathbf{w}\|_{2}^{2} \mathbf{I} & 2 \mathbf{p} \mathbf{w}^{H}-\mathbf{D}^{H} \\
2 \mathbf{w} \mathbf{p}^{H}-\mathbf{D} & \|\mathbf{p}\|_{2}^{2} \mathbf{I}
\end{array}\right]\left[\begin{array}{c}
\Delta \mathbf{p} \\
\Delta \mathbf{w}
\end{array}\right]=-\left[\begin{array}{c}
\left(\mathbf{w} \mathbf{p}^{H}-\mathbf{D}\right)^{H} \mathbf{w} \\
\left(\mathbf{w} \mathbf{p}^{H}-\mathbf{D}\right) \mathbf{p}
\end{array}\right]
$$

This can be solved for $\Delta \mathbf{w}$

$$
\Delta \mathbf{w}=-\frac{1}{\|\mathbf{p}\|_{2}^{2}}\left(\left(2 \mathbf{w p} p^{H}-\mathbf{D}\right) \Delta \mathbf{p}+\left(\mathbf{w} \mathbf{p}^{H}-\mathbf{D}\right) \mathbf{p}\right)
$$


which is substituted into the equation for

$$
\begin{gathered}
\left(\|\mathbf{w}\|_{2}^{2} \mathbf{I}-\frac{1}{\|\mathbf{p}\|_{2}^{2}}\left(2 \mathbf{w} \mathbf{p}^{H}-\mathbf{D}\right)^{H}\left(2 \mathbf{w} \mathbf{p}^{H}-\mathbf{D}\right)\right) \Delta \mathbf{p}= \\
\frac{1}{\|\mathbf{p}\|_{2}^{2}}\left(2 \mathbf{w} \mathbf{p}^{H}-\mathbf{D}\right)^{H}\left(\mathbf{w} \mathbf{p}^{H}-\mathbf{D}\right) \mathbf{p}-\left(\mathbf{w} \mathbf{p}^{H}-\mathbf{D}\right)^{H} \mathbf{w}
\end{gathered}
$$

As $\operatorname{dim}(\mathbf{p}) \ll \operatorname{dim}(\mathbf{w})$ solving this and substituting back is more efficient than solving directly for the Newton step. We don't need to store the large Hessian matrix or use sparse matrices.

Selecting the largest column of $\mathbf{D}$ as initial value for $\mathbf{w}$ and fixing the corresponding element of $\mathbf{p}$ to 1 , starting with some subspace steps and finishing with a few full Newton steps will normally converge to a solution when a wave is present in $\mathbf{D}$.

\section{References}

Keniry, J. T., Barber, G. C., TAYlor, M. P. and Welch, B. J. (2001) Digital processing of anode current signals: An opprtunity for improved cell diagnosis and control. Light Metals, pp. 1225-1232 (ISBN: 0-87339-480-1, ISSN: 1096-9586).

Mallat, S. (1998) A Wavelet Tour of Signal Processing, Academic Press, San Diego, (ISBN: 0-12-466605-1).

Romerio, M.V. and ANtille, J. (2000) The numerical approach to analyzing flow stability in the aluminium reduction cell. Aluminium, 76, 1031-1037 (ISSN: 0002-6689). 